\title{
Open Scholarship as a mechanism for the United Nations Sustainable Development Goals
}

Jonathan P. Tennant ${ }^{1}$, Wojciech Francuzik ${ }^{2}$, Daniel Dunleavy ${ }^{3}$, Benedikt Fecher ${ }^{4}$, Monica GonzalezMarquez ${ }^{5}$, Tobias Steiner 6

${ }^{1}$ Institute for Globally Distributed Open Research and Education, UK.

2 Department of Dermatology, Venereology and Allergology, Charité - Universitätsmedizin Berlin, corporate member of Freie Universität Berlin, Humboldt-Universität zu Berlin, and Berlin Institute of Health, 10117 Germany.

${ }^{3}$ College of Social Work, Florida State University, University Center C, 296 Champions Way, Tallahassee, Florida, 32304

${ }^{4}$ Alexander von Humboldt Institute for Internet and Society, Berlin, Germany.

${ }^{5}$ RWTH - Aachen University, Germany

${ }^{6}$ Community-led Open Publication Infrastructures for Monographs (COPIM), Centre for Postdigital Cultures, Coventry University, UK

One sentence summary: We are making an open call to support a global organisation and infrastructure that will strategically implement open scholarship to effectively meet the United Nations 2030 Sustainable Development Agenda.

Abstract: Traditional methods of scholarly publishing and communication are ineffective in meeting the United Nations Sustainable Development Goals (SDGs). The SARS-CoV-2 pandemic has demonstrated that, in times of need, the global research community can activate and pool its knowledge and resources to collaborate on solving problems. The use of innovative Web-based technologies, including open source software, data-sharing archives, open collaboration methods, and the liberation of thousands of relevant research articles from proprietary sources show us that the fundamental components of a fully open system are readily available, technologically efficient and cost-effective. If we are to achieve the SDGs by 2030 , systematic reform and explicit adoption of open scholarship strategies at scale is necessary. We propose that the United Nations and parallel entities take a position of leadership by creating or funding an organisation or federated alliance of organisations to implement these reforms. 
This is a preprint submitted to Policy Forum section of Science

Article 27 of the United Nations Universal Declaration on Human Rights: "Everyone has the right freely to participate in the cultural life of the community, to enjoy the arts and to share in scientific advancement and its benefits."

One month after the ongoing coronavirus SARS-CoV-2 outbreak began, on January 31, 2020, the Wellcome Trust issued a press release listing scholarly publishers who had committed to sharing research data relating to the virus. On March 13, 2020, government science leaders from around the world also called on scholarly publishers to make all relevant research publicly accessible. The current structure of our global scientific communication system, based on knowledge prohibition, is directly at odds with the fundamental human right to "share in scientific advancement and its benefits". In fact, it has been explicitly designed to violate this right. This crisis has underscored that it is long past time we placed the sovereignty of our knowledge production and dissemination systems in the commons, rightfully positioned as a human endowment, based around new kinds of organisations that can appropriately adapt to or handle the complex issues we face.

Many of the publishers who made SARS-CoV-2 literature available are simultaneously lobbying President Trump to maintain 12-month embargoes on free access to published works. This action blatantly contradicts their temporary commitment to SARS-CoV-2 research, while remaining inactive on all other societal and global health issues to which knowledge access is critical (1). Even today, most research on global health remains privatised (2), and initiatives for sharing this research during public health emergencies remain woefully under-developed (3). The fact that publishers are making knowledge about SARS-CoV-2 public means that considerable relevant information already exists, it is just not usable by those who need it. We saw it happen with Ebola in 2014 and Zika in 2016, and we are seeing it again right now. Life-saving knowledge is trapped in an anachronistic, obtuse format, locked in proprietary systems that prohibit public access and re-use by design. Some features of this system include: low research reliability, verification, and reproducibility; high exploitation of free labour and market dysfunction by for-profit entities; high epistemic and cultural inequity; and virtually zero technological innovation since the advent of the scholarly journal in the 17th Century. And yet, this system costs around USD $\$ 25.7$ billion each year to maintain (4), about $70 \%$ of which comes from public funds.

Now, more than ever, during a time of isolation, confinement, and limited mobility, validated knowledge must flow freely. The SARS-CoV-2 pandemic must serve as an urgent wake-up call to transform the capacity of our current scholarly institutes to handle the grand challenges that we collectively face. The UN has 17 Sustainable Development Goals (SDGs) with 169 targets at the heart of providing economic, socio-cultural, and ecological prosperity and peace for all. SARS-CoV-2 is one 
This is a preprint submitted to Policy Forum section of Science

small part of just one of these. The SDGs comprise a global framework for substantial public, philanthropic, and private investment, that promises to revolutionise virtually all aspects of society. Adopted by all 193 UN member states in 2015, they are supposed to be met by 2030. At their heart is a combined strategy for tackling the global climate crisis, ending poverty and social inequity, and promoting health and education around the world. Scholarly research institutes play an indispensable role in meeting the SDGs, but cannot do so if their relationship with the public remains in its dysfunctional pre- SARS-CoV-2 state.

Scholarly knowledge is a resource that, unlike many others, is infinite. If we share data, ideas, and knowledge with others, knowledge only grows. Scientific knowledge is the most sustainable resource we have. During the COVID-19 outbreak, while most of our global systems and infrastructures collapsed, our knowledge production system woke up. Researchers adopted a range of what are often called 'open scholarship practices' to combat the threat, with nations uniting to collaborate and share knowledge and resources. For example, there are more than 700 preprints on COVID-19 SARS-CoV-2 available from medRxiv and bioRxiv. "Outbreak Science Rapid PREreview" was launched as an open source platform for rapid peer-review of these preprints (5). Researchers developed an interactive Web-based dashboard to track SARS-CoV-2 in real time, and provide substantial data and resources (6). Just One Giant Lab (JOGL) is hosting a non-institutional community that is building open-source methodologies and equipment to prevent SARS-CoV-2, test for the virus and treat its symptoms. And in education, we are seeing a global wave of emerging SARS-CoV-2-related open teaching and learning materials (OER), as well as increased interest in approaches to open networked education practices and pedagogies to inform and educate pupils, students, staff, other researchers, as well as the wider public about the pandemic. These are efforts that must be supported and expanded to encompass all of science, including critical areas such as climate change and vaccine safety. Though much has been done to make scientific literature freely available using an open access paradigm, substantial concurrent efforts must be dedicated to teaching how to interpret that literature.

These are conscious choices that individuals and organisations made to be more open in order to reach a specific goal. It is difficult to see now how we can consciously return to an inefficient proprietary system after seeing the dramatically increased productivity of an open one. Yet scholarly processes, and institutes are not currently prepared to face the complexities associated with these global challenges. However, it is easier to see how a more open system can be adapted into a framework aligned with the goal-setting format of the Sustainable Agenda. The SDGs are underpinned by issues that are complex, multifaceted, and non-linear (7), and need institutes that can tackle them appropriately. They require new and united forms of leadership, strategic visions, decentralised 
This is a preprint submitted to Policy Forum section of Science

organisational structure, and coherent and effective messaging that combines scholarship in a functional way to serve society using the SDGs as the operational framework. SARS-CoV-2 pandemic highlights again how woefully ill-informed our decision-makers have been regarding matters of national and international health security, and often even complacent or ignorant to the available scientific evidence (8). If we want an informed global society working towards these issues, and setting the most urgent and fundamental priorities, we must have a robust and reliable scholarship ecosystem in place first. This ecosystem must focus on accessibility of all scientific knowledge, an infrastructure and legal framework to vastly improve re-usability of that knowledge, and an educational paradigm for scholars, decision-makers and laypeople where science literacy centers on the ability to critically question conventional wisdoms, and on the process verification aspects of science instead of downgrading science to mere fact collection.

A roadmap for Citizen Science, a fundamental part of Open Scholarship, has already been designed for integration into the SDGs (9). At least in Europe, initiatives such as the European Open Science Cloud, backed by the FAIR data principles (10), can be similarly integrated into the SDG framework. Supra-national initiatives such as the UNESCO-backed Global Alliance of Open Access Scholarly Communication Platforms are perfectly positioned to support this. The theoretical underpinnings of open scholarship have been thoroughly-documented, and perhaps best conceptualised in the 'Schools of Thought' (11). The Foundations for Open Scholarship Strategy Development was designed on top of these schools to provide a broad, international strategy for the implementation of open scholarship that meets the needs of different national and regional communities but works globally (12). It operates at the individual, group, institutional and national levels, in a time-frame that is suitable for meeting the 2030 agenda for the SDGs.

Central to all of these efforts is the development of scholarly infrastructure required to foster equitable models of participation with regards to scientific results, research processes, scholarly communication, and facilitation of education (13) in a global framework. This community-led global commons must be consciously guided by the principles of open source so as to actively counter the ongoing and widespread privatization and commercialization of critical aspects of scholarly endeavours. In addition, these tools must be strongly and deeply people centered, taking into account the way people think and use information cross-culturally, cross-linguistically and cross-disciplinarily. They must also be designed to be as minimally cumbersome and time-consuming as possible. With global scholarship systems in a more reliable state, they can help to underpin the next phase in our civilisational development. Key strategic priorities of this include removing artificial boundaries between traditional disciplinary concepts; updating our scholarly communications mechanisms to a 
This is a preprint submitted to Policy Forum section of Science

state that is fit for the digital age; developing a greater understanding of the societal value of research; and establishment of a roadmap and framework that strategically combines open scholarship with the SDGs.

Development and maintenance of this infrastructure should come from a federated network or alliance of scholarly institutes, akin to the Max Planck Society in Germany. This organisation must operate simultaneously at the political, public, and infrastructural levels. It must be held to the highest ethical standards of scientific conduct, embedding the principles, complexities, and practices associated with openness within global scholarly communities to help buttress and advance human development and civil society. A democratic, transparent, and rigorous research base, fuelled by open collaborations between civil society and research institutes, serves as a powerful instrument for influencing policy-making. With such a strategic connection between open scholarship and the SDGs, the dependency on the UN secretariat would be reduced, with developments instead from working groups, consultations, and the work of the UN's High-level Panel of Eminent Persons (HLP), which relies on an evidence-based and science-informed strategy. Through this, global scholarship will become central to addressing the deeply rooted structural causes that are the source of the problems the SDGs address, including market consolidation and manipulation, political corruption, and extreme wealth; most of which center around a failing global economic model.

Global co-operation on knowledge sharing through functional interdisciplinarity must be our highest priority. An immediate component of this infrastructure must include machine-readable inventories (14) of all relevant knowledge on all global health issues, presented in FAIR-compliant open source, non-profit virtual research environments, open to machine learning, text-mining, and Artificial Intelligence re-use. As complex global issues, they require a radical global funding vision. The choice is ours: We can invest now in the knowledge infrastructure SARS-CoV-2 outbreak has shown we desperately need and thus create the foundation for a happy, healthy, and equitable society and thriving environment. Or we can pay a much higher price in lives and financial resources when the next pandemic strikes. We believe the United Nations themselves should fund this organisation, equally through each of the responsible participating members. This decentralised, democratic, governance structure and commitment to open scholarship will be an archetypal transformation of global research systems and an essential part of realistically delivering the Sustainable Development Goals by 2030 .

\section{References}


This is a preprint submitted to Policy Forum section of Science

1. S. Day, S. Rennie, D. Luo, J. D. Tucker, Open to the public: paywalls and the public rationale for open access medical research publishing. Research Involvement and Engagement. 6, 8 (2020).

2. E. Smith, S. Haustein, P. Mongeon, F. Shu, V. Ridde, V. Larivière, Knowledge sharing in global health research - the impact, uptake and cost of open access to scholarly literature. Health Research Policy and Systems. 15, 73 (2017).

3. J.-P. Chretien, C. M. Rivers, M. A. Johansson, Make Data Sharing Routine to Prepare for Public Health Emergencies. PLOS Medicine. 13, e1002109 (2016).

4. R. Johnson, A. Watkinson, M. Mabe, The STM Report: An overview of scientific and scholarly publishing. International Association of Scientific, Technical and Medical Publishers (2018).

5. M. A. Johansson, D. Saderi, Open peer-review platform for COVID-19 preprints. Nature. 579, 29$29(2020)$.

6. E. Dong, H. Du, L. Gardner, An interactive web-based dashboard to track COVID-19 in real time. The Lancet Infectious Diseases. 0 (2020), doi:10.1016/S1473-3099(20)30120-1.

7. S. Fukuda-Parr, D. McNeill, Knowledge and Politics in Setting and Measuring the SDGs: Introduction to Special Issue. Global Policy. 10, 5-15 (2019).

8. Coronavirus: three things all governments and their science advisers must do now. Nature. 579, $319-320$ (2020).

9. S. Fritz, L. See, T. Carlson, M. (Muki) Haklay, J. L. Oliver, D. Fraisl, R. Mondardini, M. Brocklehurst, L. A. Shanley, S. Schade, U. Wehn, T. Abrate, J. Anstee, S. Arnold, M. Billot, J. Campbell, J. Espey, M. Gold, G. Hager, S. He, L. Hepburn, A. Hsu, D. Long, J. Masó, I. McCallum, M. Muniafu, I. Moorthy, M. Obersteiner, A. J. Parker, M. Weisspflug, S. West, Citizen science and the United Nations Sustainable Development Goals. Nat Sustain. 2, 922-930 (2019).

10. B. Mons, C. Neylon, J. Velterop, M. Dumontier, L. O. B. da Silva Santos, M. D. Wilkinson, Cloudy, increasingly FAIR; revisiting the FAIR Data guiding principles for the European Open Science Cloud. Information Services \& Use. 37, 49-56 (2017).

11. B. Fecher, S. Friesike, in Opening Science: The Evolving Guide on How the Internet is Changing Research, Collaboration and Scholarly Publishing, S. Bartling, S. Friesike, Eds. (Springer International Publishing, Cham, 2014; https://doi.org/10.1007/978-3-319-00026-8_2), pp. 17-47.

12. J. Tennant, J. E. Beamer, J. Bosman, B. Brembs, N. C. Chung, G. Clement, T. Crick, J. Dugan, A. Dunning, D. Eccles, A. Enkhbayar, D. Graziotin, R. Harding, J. Havemann, D. S. Katz, K. Khanal, J. N. Kjaer, T. Koder, P. Macklin, C. R. Madan, P. Masuzzo, L. Matthias, K. Mayer, D. Nichols, E. Papadopoulou, T. Pasquier, T. Ross-Hellauer, M. Schulte-Mecklenbeck, D. Sholler, T. Steiner, P. Szczesny, A. Turner, Foundations for Open Scholarship Strategy Development (2019), doi:10/gft5hx. 
This is a preprint submitted to Policy Forum section of Science

13. D. J. Dunleavy, "Coronavirus As Impetus For a Lasting Change in Research Culture" (preprint, SocArXiv, 2020), doi:10.31235/osf.io/2ryt3.

14. M. Gonzalez-Marquez, N. Dutra, S. Tsuji, C. Bergmann, Meta-Data Inventory Project description (in progress). (2020) 10.13140/RG.2.2.31714.48323.

\section{Acknowledgements}

To all of those working tirelessly on helping to combat the coronavirus threat, you have our deepest gratitude. We would like to thank Surya Dalimunthe, Serge Bauin, and Thomas Landrain for input into this manuscript. 